\title{
APPLICATION OF ARTIFICIAL INTELLIGENCE IN ALGORITHMIC TRADING
}

\author{
Rohan Pothumsetty \\ Department of Commerce \\ Christ University, Bengaluru, Karnataka
}

\begin{abstract}
In the present era there are many challenges affiliated with carrying out algorithmic trading. Regardless of these challenges, most of the stock trading companies in the world are trying to switch from discretionary trading to algorithmic trading. Algorithmic trading refers to trading tactics that are automatic both in terms of executing and identifying trades. Algorithmic trading is one of the most in demand technology in the current era. Algorithmic trading has given stock trading firms more potential in the swiftly developing markets by removing human errors and completely transforming the way stock markets are interconnected today. This research paper talks about how algorithmic trading works using Artificial intelligence technology and discusses the top five trading strategies adopted in algorithmic trading and the key advantages of implementing them. This research paper also emphasizes on evaluating the critical differences between discretionary trading and algorithmic trading and why traditional traders should consider switching to algorithmic trading platforms.
\end{abstract}

Keywords - Artificial Intelligence, Algorithmic trading, Discretionary trading, Technology

\section{INTRODUCTION}

Technology has transfigured the way stock markets perform, and the way investment assets are actively traded. The two most consequential technological innovations are financial investors manoeuvring computer systems to mechanize their stock trading processes and the financial markets restructuring itself so virtually that all the markets are right now limit order books (JAIN, 2005). Consequently, in the finance world, the emanation of the internet and its consequent technological innovations are significantly influencing the way financial markets work and function. For example, most of the financial transactions in the present age have become electronic, and the total period it takes to expedite a stock trade has significantly reduced to nanoseconds. Algorithmic trading is the practice of purchasing or trading security established to some preprescribed set of rules tested on past or historical data ("The Future of Algorithmic Trading"n.d, 2017). These sets of rules are based on charts, indicators, technical analysis, or stock essentials. For instance, suppose you have a proposition to purchase a particular stock assuming that the stock will end up in losses for 5 consecutive days, In this case, you can write and design an algorithm in such a way that you buy order for the particular stock is met when all the conditions are pacified. The future of algo-trading mainly instigates with resource allocation, which is one of the main factors which affects the stock's overall performance. Algorithmic trading has propagated substantially over the past ten years. In the USA'S stock trading market, about 70 per-cent of the comprehensive trading volume is initiated through algorithmic trading. The total trading volume of Algo-trading, especially in a developing economy like India, is at around 40 per-cent. The recent report by Forbes evaluated that the total world market for algorithmic trading is said to expand by 10.3 per-cent by the year 2020. Currently, most of the regulators and regular stock market investors have moved in the direction of Highfrequency trading (HFT) and algo-trading. High-frequency trading (HFT) is a category of algorithmic trading where vast volumes of stocks and shares are sold and bought mechanically at very high speeds. High-frequency trading (HFT) will tend to develop continuously and will become the most authoritative form of algorithmic trading in the future. Algorithmic trading is completely reforming the way trading is done. Stock traders are using algorithms to bring more speed and efficiency to financial trading markets. In the forthcoming days, we will perceive a very high level of automation, especially in trading markets. The algorithms that are developed will tend to become more complicated as it will be able to accommodate themselves to diverse trading patterns using Artificial Intelligence (AI). We can also anticipate algotrading to move into more pragmatic Machine learning (ML) dexterity that can manage real-time deciphering of large volumes of data from many different sources.

\section{RESEARCH GAP}

Most of the Traditional traders, especially in a developing economy in India are not aware of algorithmic trading this research paper aims to fill this gap and to create awareness about the rise of algorithmic trading and why people who actively trade in the stock market should consider switching to algorithmic trading. This research paper also focuses on the key benefits traditional traders receive in the process of 
switching from discretionary trading methods to algorithmic trading.

\section{RESEARCH METHODOLOGY}

This research paper is based on secondary data, as there is no primary data research conducted in the field of Artificial Intelligence $(\mathrm{AI})$ and algorithmic trading. Pervasive research was regulated to pick out articles from ProQuest and Google scholar. Secondary data was also collected from different grey literature sources such as magazines, websites, articles, and blog posts.

\section{RESEARCH OBJECTIVES}

- To study how algorithmic trading works using Artificial Intelligence (AI)

- Analysis of top five trading strategies adopted in algorithmic trading

- Critical differences between discretionary trading and algorithmic trading and why traditional traders should consider switching to algorithmic trading

\section{RESULTS AND DISCUSSION}

\subsection{How Algorithmic trading works using Artificial Intelligence:}

Technological amendments have entirely changed the way financial instruments are traded in the stock markets. Every single step in the stock trading process from entry order to trading venue to the back office is all highly digitalized, substantially minimizing the total cost by different intermediaries (Hendershott, Jones \& Menkveld, 2011). By diminishing the total costs of stock trading, Artificial Intelligence (AI) has the inherent capacity to authorize more compelling risk sharing, improve liquidity, and also make the price more dynamic. This will eventually abbreviate the firm's total cost of capital incurred. Algorithmic trading or Algotrading is a striking representation of this advanced technological change. Many stock market players are now adopting algorithmic trading in their stock trading mechanisms. Algo-trading is defined as the use of computergenerated algorithms to mechanically make individual stock trading decisions, submitting orders, and finally managing those orders after submission (Hendershott, Jones \& Menkveld, 2011). In the present era, most of the large stockbroking agencies will tend to offer a collection of algorithms to its clients to help them validate orders in pairs of stock, in a basket of stocks, or just a single stock (Hendershott, Jones \& Menkveld, 2011). Before the introduction of electronic trading system trading stocks was a paper-based activity. And one needed to be attending in-person to purchase new stocks or sell them. After the introduction of the Dematerialization (DEMAT) account, most of the paper-based certificates were being reinstated with electronic-based certificates as they could be quickly registered online. It intensified the total variation in the prices of the stocks as the trading procedure was quicker. With the introduction of algorithmic trading, you can design an algorithm that will command the computer to sell or buy individual stocks when predefined conditions are met (Moy das, 2019). Algorithmic trading can help to trade at a very high speed and recurrence, which is impossible for a human trader to carry out. Algorithmic trading platforms usually consist of four different main components that are the trade execution handler, data handler, The model component, and the strategy. The four main components which are used to run the algo-trading platform are discussed in detail below.

\subsubsection{The model component of Algorithmic trading:}

The model component of Algorithmic trading is the depiction of the outside world as the algorithmic trading system perceives it. The financial models that are developed usually tend to constitute how the algo-trading system considers the market works (Moy das, 2019). The fundamental goal of these financial models is to create interpretations about the world and, in this case, the financial markets. Financial markets can be structured using numerous methods and techniques. Still, all the financial models are doing just one thing, depleting a complicated system into a manageable set of rules which illustrates the behaviour of that system under a different sequence of events. The conventional methods include decision trees, frizzy logic techniques, neural networks, mathematical models, and induction rule sets.

The practice of employing mathematical models to illustrate the performance of stock markets is known as quantitative Finance. Most of the models in quantitative finance work on the intrinsic presumption that the market prices and the return advance over time in conformity to a stochastic process, which means that stock markets are very random. This is a very useful presumption which is at the disposition of all the derivatives pricing models and other security valuation models. Fundamentally most of the quantitative financial models remonstrate that the returns of any given financial security are persuaded by one or more market risk circumstances. The level to which these risk factors emancipate market returns is known as price sensitivity. For instance, a well-assorted portfolio's market returns may be determined by the fluctuations in foreign exchange rates, Markets short-term rates, and the returns in the stock market. These factors can be calculated historically and can be used to develop a model that reproduces what those risk factors can do and can predict what the returns on that particular portfolio might be.

The fuzzy logic system has been extensively used in robotics, home appliances, machinery, expert systems. Lately, the application of the fuzzy logic model in the field of algorithmic trading and Finance has also been reported (W. Cheung \& U. Kaymak, 2020). The fuzzy logic model is a procedure for variable processing that allows numerous values to be formed through the same variable (Scott, 2019). The fuzzy logic 


\section{International Journal of Engineering Applied Sciences and Technology, 2020 Vol. 4, Issue 12, ISSN No. 2455-2143, Pages 140-149 \\ Published Online April 2020 in IJEAST (http://www.ijeast.com)}

model in algorithmic trading is constructed to solve problems by taking into account all the accessible information and making the best practical decision given the input. The notion of fuzzy logic is the principal constituent to the programming of Artificial intelligence solutions. Artificial intelligence systems continue to grow in the financial system across various range of sectors as the programming competence of fuzzy logic also diversifies. IBM's Watson is the most renowned AI system using deviations of fuzzy semantics and fuzzy logic models. Notably, in algorithmic trading, fuzzy logic is being used in the Machine learning (ML) system advocating the productivity of investment intelligence. In some of the most advanced algorithmic trading model's fuzzy logic models can help trading analysts create automatic sell and buy signals. These models help investors to reciprocate to a wide array of adjusting market variables that influences their financial investments. In some of the other advanced algorithmic trading models, can utilize a large number of securities in real-time and give the investor the best obtainable chance. Fuzzy models are frequently used when a stock trader strives to make use of numerous factors for contemplation. This can result in a confined analysis for stock trading decisions. Stock traders also can program a mixture of rules for authorizing trades. For instance, if the moving averages are very low and the relative strength index is low, the algorithm will automatically sell the stocks, and if the moving averages are high and the relative strength is also high, the algorithm will automatically purchase stocks. Thus, fuzzy logic models help a stock trader to program their own trading algorithm in low and high and also buy and sell stocks automatically. Fuzzy logic models also help to decipher stock trading by reducing the total risk involved with human-based emotions and accurately influencing data through facts and figures ("What is the Role of Fuzzy Logic in Algorithmic Trading? GeeksforGeeks"n.d, 2020).

The symbolic logic is a form of reasoning which fundamentally includes the estimation of predicates (These are logical statements fabricated from logical operators such as AND, XOR, and OR) to be either false or true (Scott, 2019). Whereas, the fuzzy logic model moderates the binary true or false obligation and enables any given predicate to affiliate to the set of true or false predicates to contrasting degrees. About stock markets, the feeding of data into the technology may include pointers which are anticipated to correlate with the total returns of any given security. These indicators may be technical, fundamental, or quantitative. For instance, the fuzzy logic model might extrapolate from past data if the five days weighted moving average is more significant than or equal to the ten-day weighted moving average then, in this case, there is a 65 per-cent chance that price of the stocks will rise over the next five days.

A decision tree trading model is a linked acrylic graph with the leaves designated by decisions ("Decision Tree Classifier For Trading Part-1"n.d, 2017). The procedure of regulating decisions starts from the root node, then relying on the node value the progression into either the right node or the left node takes place. Due to this, the leaf node at the end is taken that is an order to purchase a particular stock and open a long position or sell out the acquired volume ("Decision Tree Classifier for Trading Part-1"n.d, 2017). The size of the position is decided by the highest conceivable number of shares that can be bought at the present volume. Decision tree models are quite equivalent to induction models excluding that the rules are structures in the shape of a tree. In the field of computer science, a kind of data structure in which every single node has at least two children is known as the right child and the left child. In this context, each node designates a decision tree, and each of the child nodes is a terminal node or a division boundary, which signifies an output. Presently, two types of decision trees are significantly used in algorithmic trading that is the regression trees and classification trees. Regression trees incorporate outcome variables for a particular variable. Whereas, classification trees comprise different classes in their outputs (Ex- Hold, buy, or sell). The category of data that is used to instruct the decision tree will influence what type of decision tree is generated. The significant algorithms used for generating decision trees are genetic programming and C4.5. In the case of rule induction, the information into a decision tree model may contain quantities for a given set of technical, statistical, or fundamental factors which are considered to drive the return of securities.

Neural network models are one of the acclaimed Machine learning (ML) model that is convenient for algorithmic traders. Neural network models usually are composed of layers of interrelated nodes between outputs and inputs. In Neural network models, the individual nodes are called perceptron's and are similar to a multiple linear regression excluding that they feed into something called activation function, which sometimes may or may not be non-linear (Moy das, 2019). In the case of non-concurrent neural networks, the perceptions are organized into many different layers and are inter-connected with each other. There are three different types of layers in neural networks; they are the output layer, the input layer, and the hidden layer. The output layer usually contains sell, hold or buy classifications and binned returns. Whereas the input layers derive the normalized inputs, which would be the various factors presumed to drive the various returns of the security. The hidden layers are used to accommodate the weightings on output and input layers until the total error of the Neural network is reduced. One explanation to this is that the hidden layers derive essential features in the data which have predictive power with regard to the outputs. In extension to these models, there are a number of other decision-making models that can be used in the setting of algorithmic trading to help quantitative traders to make forecast the changes in the price of the security. The selection of the model has a direct relationship with the interpretation of the algorithmic trading systems. Using multiple different models has improved the accuracy of prediction that will maximize the intricacy of programming 


\section{International Journal of Engineering Applied Sciences and Technology, 2020 \\ Vol. 4, Issue 12, ISSN No. 2455-2143, Pages 140-149 \\ Published Online April 2020 in IJEAST (http://www.ijeast.com)}

performance. To make the trading system more intelligent, the system should store any kind of data concerning any mistakes made in the past and should adjust to its internal models according to those specific changes.

\subsubsection{The data component of Algorithmic trading:}

The influence Big data is creating in the finance industry is more of a splash than a ripple (Eastwood, 2017). The technology is ascending at a dramatic rate, and the outcomes are far-reaching (Eastwood, 2017). Maximizing complicatedness and data generation is changing the way financial industries operate. At present, the world is generating about 2.5 quintillion bytes of data every single day, and this denotes an inimitable opportunity for analysing, leveraging, and processing the information in many useful ways. Algorithms and Machine learning (ML) are exponentially being used in stock trading to evaluate a massive collection of data and make decisions that human beings cannot. The trading industry usually depends on accurate inputs. Previously, numbers were calculated by human beings, and outcomes were drawn from deliberated risk and trends. In the present age, the functions are performed by Artificial intelligence systems. The AI systems can compute numbers from large databases and provide precise solutions instantly. The data component in financial trading does not just calculate fluctuations and changes in price; it also tries to comprehend the various reasons for these price fluctuations (Matthews, 2019). The process of data collection makes way for various predictive models. Data models can start identifying trends while Machine learning models can trace patterns which human beings might miss. The use of data models in trading helps investors and financial traders make smarter decisions. Algorithmic trading systems can utilize both Structured and Unstructured data. Data is said to be structured if it is arranged in accordance with some pre-arranged structure. Some examples of structured data include XML files, JSON files, Spreadsheets, data structures, and databases. Also, data related to markets such as the end of the day prices, Inter-day prices, and market-related data are generally accessible in a structured format. The data is said to be unstructured if it is not arranged according to any pre-arranged structure. Examples of unstructured data include social media, news audio; this type of data structure is intrinsically more complicated to process and frequently needs data mining and data analytics to examine and derive useful inferences from it. For instance, in the case of unstructured data from news and social media, Quantitative traders tend to use sentimental analysis to derive useful information from unstructured data. Sentimental analysis refers to the use of Natural language processing (NLP), computational linguistics, and text analysis to influence personal information or the passionate state of the subject/writer or topic (Yip, 2018). It is generally used in reviews that liberate businesses much time from physically reading any comments (Yip, 2018).
5.1.3 The execution component of Algorithmic trading: Algorithmic trading is wholly determined on the high speeds at which financial institutions can get their particular order to the market to the market ("Algorithmic trading using technology to speed things up"n.d, 2020). With more than thousands of traders administered in seconds. The use of the arbitrage trading model could encode into lots of profits. Most of the algorithmic traders invest much money to minimize the total time it takes to execute a particular trade. Brokers simultaneously invest in computing and processing power and also use the best talent available in the market to create the best trading algorithms. The key to the algorithmic trading market is the speed of the network that they are trading on. The most significant feature is the Networks latency, which is also known as the "Speed factor" in algorithmic trading. Algorithmic trading strategies that idealize the value of Highfrequency trading (HFT) are immensely reliant on Ultra-low latency (Goldstein, 2014). Ultra-low latency is concerned with trading at a speed of less than one microsecond. To earn profits, quantitative traders must continuously enhance their systems to have a competitive edge. High-frequency traders must also use Ultra-low latency to execute thousands of stock trades before an investor even knows what is going on (Goldstein, 2014). The execution component in algorithmic trading requires to meet both the functional and non-functional conditions of the algorithmic trading platforms. For instance, the recurrence at which trades are executed, the speed of execution of the trade. Any enforcement of the algorithmic trading system must be able to discharge those stipulations.

\subsubsection{The monitor component of Algorithmic trading:}

Artificial Intelligence systems comprehend mainly using objective functions. Objective functions are mainly the mathematical functions that calculate the interpretation of the algorithmic trading system. Regarding Finance, measures of risk-adjusted returns such as Sortino ratio, Treynor's Ratio, and Sharpe's ratio. The model component in algorithmic trading platforms would be proposed to enhance one or more of these quantities. The problem with this is that stock markets are vigorous. In this case, the trading models, Neural networks, and logics which worked before might stop functioning over some time. To content this, the algo-trading should improve the stock trading models with particulars about the models themselves. This will help the models to adjust themselves to changing market environments.

\subsection{Analysis of top five trading strategies in Algorithmic trading:}

\subsubsection{Arbitrage trading strategy:}

The arbitrage trading strategy strives profits from short-term market inefficiencies that bring about the mispricing of the same asset in different markets or related assets in the same financial market (Maverick, 2019). Arbitrage trading strategy quickly asserts to rectify such temporary pricing inefficiencies 
in the market and to get prices correctly back in the line transversely across various markets. The provisional imbalances that form the possibility to make the coinciding buy and sell trades, that lock in a small profit was deriving from changes in pricing (Maverick, 2019). For instance, in the stock market, there may be a negligible inconsistency between the Euro and the British Pound and two other pairs of currencies US dollars and British Pounds that can authorize the stock traders to gain profit from synchronically selling the Euro/US dollar and purchasing Britain pounds/US dollar and Euro/ Britain pounds. Considering that arbitrage trading's opportunities and chance last for a brief period sometimes for a few seconds, and it also takes much time for quantitative traders to perform arbitrage calculations on their own. So, most of the quantitative traders tend to use a wide range of software programs that can immediately identify and enumerate various arbitrage opportunities. One kind of software engaged by traders is automated trading software. This software is packed onto a traders trading platform, and when the software ascertains an arbitrage opportunity, it instantaneously instigates the selected trades on behalf of the quantitative trader. This software is primarily designed to prevail one of the principal challenges of arbitrage trading the accurate and timely trade investment necessary to take precedence of trading vantages, which may last only for a few seconds. Quantitative traders who are not convenient with trades being instinctively executed and preferring to make all the trading decisions on their own tend to use trade alert software. Similar to automated trading software's trade alert, computer software interminably examines different instruments, brokers, and markets for different arbitrage trade opportunities. When the software perceives an arbitrage opportunity instead of executing the trade mechanically, the software notifies the trade about the arbitrage opportunity, who then makes the verdict whether or not to carry out the trade on the opportunity. Some of the quantitative traders, instead of utilizing their software detection program, advocate a remote alert service. Signing up to this service enables them to receive arbitrage trading opportunity notifications in the same manner as using their arbitrage detection software program, the critical difference is that the alert notifications are given by software working outside, or another location of the trader's own network. Quantitative traders can also utilize an automated trading system to their benefit along with arbitrage strategy. The automated trading systems usually depend on algorithms to detect price inconsistencies; as a consequence, they enable a stock trader to jump on a change or exploit in the financial markets before it becomes common knowledge, and the markets go back to normal ("Arbitrage trading in forex explained"n.d, 2020). The key advantages of implementing an arbitrage trading strategy are depicted below. The arbitrage trading strategy is one of the trading strategies that has been deliberated the most productive to make profits and also the most innocuous way to invest ("Benefits of an arbitrage trading system | executium", 2020). Arbitrage trading strategy has been able to assure perpetual profits to the traders using this strategy. The traders who trade on arbitrage use the strategy of trading in huge volumes and trading as persistently as possible. This is for the reason that price discrepancies are very marginal, and therefore to get a specific amount of return, you have to invest more and frequently ("Benefits of an arbitrage trading system | executium", 2020). Arbitrage trading has guaranteed constant cash flow and a considerable amount of profits to traders.

The arbitrage trading strategy eventually has no risk. This is because there is no disclosure of any cash, and the profits are achieved in a short period. The arbitrage trading software systems ensure that they identify any price variation and take benefit of the short period to make profits. Thus, there is no risk involved in losing your money.

To take precedence of the price changes, there must be compelling software systems that can be used to detect any price fluctuations within a brief period, practically seconds. This validates quick manipulation of the prices as the software systems have been calibrated to do intricate mathematics to easy and fast detection of arbitrage opportunities.

Distinct from the open market, the arbitrage strategy is very innocuous and risk-free to invest your money. You don't have to be distressed over profits as the trade guarantees a perpetual gain of funds you have invested. The trading algorithm has assured many sellers a very effortless and most adequate way of making money.

\subsubsection{Technical analysis trading strategy:}

Technical analysis strategy is the predicting of future price activity based on an analysis of preceding price movements. Just like weather forecasting technical analysis strategy does not result in definite predictions about the future. Alternatively, Technical analysis can help traders and investors predict what is expected to happen to prices over time. Technical analysis uses an extensive assortment of charts and figures to show price changes over time. Technical analysis strategy is relevant to commodities, stocks, Indices and futures or any financial instrument which is impacted by the forces of supply and demand (Moy das, 2019). The price data in technical analysis strategy refers to any consortium of high, volume, open, close or open interest for a particular security over a particular time period. The time-period can be dependent on daily, weekly, intra-day or monthly price data and last a few years or many years. Technical analysis strategy is applicable to securities where the price is only affected by supply and demand. Technical analysis does not function properly when other forces control the price of the security. Technical analysis strategy is based on the following assumptions

High liquidity is fundamentally volume. Stocks which are traded heavily enables investors and traders to trade rapidly without abruptly restructuring the price of the stock. Stocks that are traded thinly are more arduous to trade as there are not many buyers and sellers at the given time. So, sellers and 


\section{International Journal of Engineering Applied Sciences and Technology, 2020 Vol. 4, Issue 12, ISSN No. 2455-2143, Pages 140-149 \\ Published Online April 2020 in IJEAST (http://www.ijeast.com)}

buyers may have to reform the accurate price substantially to make a trade. Adding to this low liquidity stocks are frequently priced low which means that their share prices can easily influenced by investors. So, thinly traded stocks are not suitable to Technical analysis.

There are no artificial price changes that is dividend, splits and distribution are the most common delinquent for simulated price variations. Artificial price changes can significantly affect the price chart and make Technical analysis arduous to apply. This kind of price influence from outside sources can easily be reported by changing the past data prior to price change.

Technical analysis strategy cannot forecast any extreme events comprising business events such as $\mathrm{CFO}$ of a company dying accidentally and also various political events such as terrorist attacks. When external forces like these are manipulating the price, investors have to wait persistently until the charts go back to normal. It is very significant to establish whether or not security meets these three assumptions before application of Technical analysis strategy. In the earlier days, Dow Jones theory laid the foundations for what later became modern technical analysis according, to technical analysis price discounts everything. This principle is equivalent to a semistrong and strong form of market efficiency. Technical analysts assume that the present price ultimately portrays all information because all the data is already exhibited in the price it denotes the fair value and should form the foundation for analysis. Since, the market reflects the expertise of all participants, including investors, portfolio managers, technical analysts and many more. Technical analysis uses the data encapsulated by the price to decipher what the market is saying with the purpose of structuring a view on the future. Most of the price movements are not entirely random; most technical analysts accept that prices trend. Sometimes there are also periods when prices do not trend. If prices were random most of the time, it would be difficult to make money from technical analysis. The key advantages of implementing Technical analysis is discussed below.

The most significant advantage of technical analysis is that it can be relevant to any financial trading instrument and in any period ("Online Trading | Forex, Spread Betting \& CFD Trading | City Index UK"n.d, 2020). A technical analysis strategy can be used to evaluate anything from commodities, stocks, forex, and interest rates. Technical analysis can be applied from a short-term aspect to a long-term period. It is also significant to understand that technical analysis strategy can be used as a standalone method or can be integrated with other methods such as market timing or fundamental analysis, The duration of the commodity market trend is recognized and monitored using technical analysis. The downward trend, sideways trend, and uptrend can be identified using technical analysis. These trends are convenient for making decisions when we invest and trade in commodity markets.

The fundamental analysis presents only one approach, which is real-life information. Whereas technical analysis indicators present a rich collection to choose from, such as volumes, oscillators, and trend indicators, provide a privilege to customize the chosen method as per the quantitative trader's needs ("Main Advantages of Fundamental and Technical Analysis | FXlinked"n.d, 2018). The quantitative trader can choose the indicators that he is comfortable with and understands the best.

Technical analysis allows the investor to create his own technical programmable mathematical models so that the quantitative trader can design his trading algorithms to suit his needs and benefits ("Top Five Benefits of Technical Analysis in Currency Trading"n.d, 2020).

\subsubsection{Momentum and trend trading strategy:}

Momentum and trend strategy are commonly used to entrap strong moves in short time periods usually for not more than a few days or a few hours. Momentum traders are emphasized on only a few trades at a time and most investigate a large watch list for a few signals (Burns, 2020). The trend followers by distinction administer portfolios for entries and exits across a variety of assets reciprocating to signals that allow them to earn profits in the long-term trends and be on the right side of unusual outlier events that direct to large profits through enormous parabolic curves in one direction over a very long period. Momentum is defined as the increase in the price of the stock that can be due to sentiment, earnings and the news. Momentum traders will take a short or long position in the stock in the hope that its momentum will proceed in either a downward or upward course in the time period they are trading. Momentum and trend technique depend on the shortterm movements in a stock's price rather than long term assessment. The most important tools used in momentum and trend strategy are discussed below.

Among all the tools known to trend traders, the moving average tool is very well-known among amateurs and professional stock traders. The moving average technique is a comprehensive tool that levels out price data by establishing a interminably updated average price. The average is taken over a particular period of time like 30 weeks, 20 minutes or any time frame the quantitative trader chooses. A moving average strategy will help to cut down the quantity of noise on a price chart (Mitchell, 2020). Moving average is mostly applied to derivative prices, trading volume, yields and percentage returns. The price of the securities will be inclined to change quickly. Moving averages helps to level out the changes helping traders and analysts to forecast the movement in the securities price (Singh, 2017). Larger subsets for enumerating moving averages will produce smoother curves and include lesser fluctuations overall.

Candle stick tool is a technique of financial chart used to portray low, high, closing and opening price changes of a security, derivative or currency for a particular period. It is similar to the shape of a candlestick thus the name (Bhagat, 2020). Candlesticks show that movement by visually designating the size of the price moves with contrasting 


\section{International Journal of Engineering Applied Sciences and Technology, 2020 Vol. 4, Issue 12, ISSN No. 2455-2143, Pages 140-149 \\ Published Online April 2020 in IJEAST (http://www.ijeast.com)}

colours. Quantitative traders use candlestick price chart to make trading decisions established on regularly occurring patterns and helps to forecast the short-term path of the price (Mitchell, 2020) . Candle stick charts helps the traders to see the direction of the market more effortlessly. The colour and shapes on the candlesticks help quantitative traders establish if an uptrend is part of a Bullish or simply a bearish spike ("Japanese Candlesticks Charts | Benefits of Candlesticks Charts |FOREX.com"n.d, 2020).

The Relative strength Index (RSI) is a momentum indicator that evaluates the consequence of recent price changes to calculate oversold or overbought situations in the price of a particular stock or asset. The RSI is usually exhibited as a Oscillator and can have a reading from 0 to 100 (Rao, 2020). The RSI divergence is calculated using the formula RSI=100(100/1+Average gain/Average loss). The average loss or gain used in the computation is the average percentage gain or losses during a look-back period. The formula tends to use positive values for any average losses. Conventional analysis and usage of RSI are the values of 70 or above denote that a security is turning to be overvalued or overbought and may be primed for a corrective pullback or trend reversal in price. An RSI below 30 denotes an undervalued or oversold situation. The key advantages of Momentum and trend trading strategy are discussed below.

Momentum and trend indicators generally show the changes of price over time and also how potent their movements will be heedless of the direction the price moves up or down.

Momentum and trend indicators are also particularly useful as they help analysts and traders to identify points where the market will reverse ("An Introduction to Momentum and Trend Trading"n.d, 2018). These points are perceived through difference between momentum and price movement.

Since, Momentum Indicators show the relative strength of price movements but assign out the directionality of the price movements then such indexes are best employed in combination with other technical indicators such as moving averages and trend lines which shows directions and price trends.

\subsubsection{Statistical arbitrage trading strategy:}

The Statistical Arbitrage was introduced in the 1980s from the hedging request formulated by Morgan Stanley's equity block trading desk operations (Kuepper, 2018). Morgan Stanley was able to evade price penalties affiliated with large block purchases by buying shares in closely associated stocks as a protection against its position, for instance, if a firm bought arbitrage block of shares it would then short a closely affiliated stock to protect against any significant decline in the market. This successfully removed any market risks while the firm risks to place the stock it had bought in a black transaction. There are many different types of Statistical Arbitrage in Algorithmic trading but these types discussed below have a more effective market place in the present age.
Risk arbitrage is a type of Statistical arbitrage that looks for profits from mergers. Algorithmic traders buy stock in the target and synchronically short the stock of the acquirer. The consequence is a profit achieved from the discrepancy between the market price and the buyout price. Unlike conventional statistical arbitrage, risk arbitrage assumes taking on some risks. The biggest risk is that the merger will fall through and the target's stock will go down to its pre-merger levels. The key to success is risk arbitrage is governing the likelihood and appropriateness of the merger and correlating that with the variation in price between the buyout offer and target stock. Some risk intermediaries have started to contemplate on takeover targets as well, which can lead to fundamentally higher profits with fairly higher risks.

Volatility arbitrage is a conventional kind of statistical arbitrage that emphasizes on taking benefit of the differences between the implicit volatility of an option and an estimation of the future accomplished volatility in a data-neutral portfolio. Fundamentally, Volatility agents are contemplating on the volatility of the primary security rather than making a directional bet on the securities price. Once a volatility agent has evaluated the future perceived volatility, he can start to look for options where the suggested volatility is either considerably lower or higher than the forecast realized volatility for the intrinsic security. If the implied volatility is lower the trader can purchase the option and protect with the underlying security to produce a delta-neutral portfolio. The quantitative trader will earn a profit on the trade when the inherent securities realized volatility moves closer to the trader's prediction than it is to markets prediction. The profit is earned from the trade through the continual reprotection needed to preserve the portfolio as delta-neutral.

High-frequency trading is quite a new advancement that extends to capitalize on the capacity of computer software systems to swiftly execute transactions. Investing in the trading sector has increased remarkably over the decade so because of this, there are many software programs that are able to complete thousands of trades per second. At the present most statistical arbitrage chances are constrained due to competition, the capacity to swiftly carry out trades is the only way to earn profits. Exponentially complex statistical models and neural networks integrated with computer software that is able to calculate numbers and perform trades faster is the key to profits for Quantitative traders. Statistical arbitrage plays a crucial role in understanding much of the day to day liquidity in the markets. It allows large block traders to place their trades without perceptibly influencing the market prices, the key advantages of Statistical arbitrage in Algorithmic trading are discussed below.

Statistical arbitrage is significant to the market and all the online quantitative traders associated. This is because whether your stock is not performing or doing well there is a place for you when Statistical arbitrage is employed. For the stocks which are performing well, you can sell your stock when the stock or product is performing well therefore in this case 
everyone wins ("7 Reasons Statistical Arbitrage is important in Online Trading | executium Trading System"n.d, 2020).

The key benefit to statistical arbitrage is that it can be used by any quantitative trader who has an online existence. For instance, a shoe seller online could benefit if he dedicated his knowledge by contrasting with other brands with a huge discrepancy in pricing. By efficiently perceiving opportunities to buy underperforming stocks line is a situation which guarantees profits.

Statistical arbitrage helps to stop future loss, this strategy is relevant to the company which is not doing well in the current market. As the data shows that the company's product is certain to diminish in the future, he is in a good position to sell his product when it is still doing well. This will significantly decrease the massive risk of a decline in the near future.

\subsubsection{Mean reversion trading strategy:}

Mean reversion strategy is a principle used in algorithmic trading that advocates that asset prices and past returns will ultimately return to the average level of the entire data set or the long-run mean (Chen, 2019). This mean can relate to another appropriate average such as average return or economic growth of an industry. Thus, mean reversion strategy has led to many investing techniques that incorporate the sale or purchase of securities or stocks whose latest performance varied greatly from their historical average. Nevertheless, a change in returns could also be a sign that a company no longer has the same probability it once did in which case there is a very less chance mean reversion would occur. A reversion to the mean incriminates any condition back to a previous state. In the case of mean-reversion if any price departs from the long-term norm will again return regressing to its understood state. The principle is concentrated on the reversion of only comparatively extreme changes as normal growth or other changes are an anticipated part of the model. Mean reversion strategy is used as a part of a statistical analysis of market conditions that can part of a comprehensive trading strategy. It commits well to the ideas of purchasing low and selling high by the yearning to discern abnormal activity that will conceptually go back to its preceding stage. Mean reversion strategy is also being used in options pricing to portray the observation that the variability of an asset will change around some long-term average. Mean reversion strategy tries to take advantage of exceptional changes in the pricing of specific security presuming that it will relapse back to its previous stage. Mean reversion strategy can be applied to both selling and previous buying as it allows a quantitative trader to profit on abrupt upswings and save on peculiar lows. Regardless, the return to a usual pattern is not assured as unpredictable highs or lows could denote a change in the norm. Such incidents could comprise but are not limited to new products releases or developments on the positive side or lawsuits and recalls on the negative side. An asset could encounter a mean reversion strategy even in the most intense event. But with most market activity there are few assurances about how specific events will or will not affect the overall appeal of specific securities (Chen, 2019). The key advantages of implement mean reversion strategy in Algorithmic trading are discussed below.

Mean reversion strategy provides flexibility this type of trading does not need you to be located or seated in front of the system the whole day. You can organize a list of stocks the day before or just earlier when the market closes and then place the order (Upadhyay, 2019) .

Mean reversion strategy provides a remarkable diversification for your long-term investments which is generally relevant to long-term trends in the market. Both the techniques have very low correlation with each other. Assorting your portfolio into non-correlated techniques will increase the risk-adjusted returns.

Mean reversion strategy provides extra returns on your preceding portfolio. If you already have an existing portfolio you can employ a mean reversion strategy to gain additional returns. You can take conventional leverage on your portfolio and enhance your returns with lesser risk.

\subsection{Critical differences between Algorithmic trading and Discretionary trading:}

Discretionary trading strategy generally has a pre-defined set of rules that they tend to comprehend throughout their trading. These set of rules are reorganized or restored based on their knowledge and what strategies work best for the discretionary trader. Some of the discretionary traders follow these rules meticulously, while others are inclined to investigate until the time, they feel that they have deciphered the code and proceed to make required adjustments in their strategy. A discretionary trader analysis the charts and signals and then makes a recommendation on whether to sell or buy the asset. Algorithmic traders use algorithms to make trading-based decisions or forecast their best chance of making a profit out of the investments they make (Thapar, 2017). The algorithms are altered based on the market scenario the type of markets, shares. An algorithmic trader chooses to make forecasts based on historical data to build an algorithmic trading strategy that matches the current market conditions. Algorithmic traders supervise the performance of the algorithms established on the logic that has been built and makes the essential changes once the developed algorithm has stopped functioning as usual. The critical differences between algorithmic trading and discretionary trading are discussed below.

One of the key differences between algorithmic trading and discretionary trading is the kind of trading strategy used. The trading strategy of discretionary traders is extracted from market conditions, learning charts, comprehending indicative signals and other associated factors which keep them to outline a specific set of rules to follow before putting an order or when to exit. Whereas, an algorithmic trader's decisions are based on an understanding of derivatives, statistics and probability, historical data and various risk management skills. This is performed by algorithmic traders and they analyse the 
market and make a judgement based on the logic set for algorithms.

Discretionary traders are vulnerable to be dominated by human emotional factors at the time of making decisions. Discretionary traders often are inclined to defend their emotional distortion at the time of determining the outcome which may lead to consequential losses. The risk of getting dominated by human emotional factors is not present in algorithmic trading. The algorithms and quantitative models are reliant on the set of instructions provided by the algorithmic trader and tend to remove any kind of human based-emotions.

Discretionary trading limits the use of automated trading systems that takes the decisions for you. It is controlled physically by the discretionary trader. An algorithmic trader does not have to manually monitor the markets and charts and trades are done mechanically. The information transfused into the software is developed by the black box and recommendations are made for the best probable outcome.

There are no pre-arranged set of rules for a discretionary trader. The exit or purchase is made established on the experience and the research done by the trader which may end up in numerous trading rules for each trade execution. The statute in algorithmic trading is pre-set and backlisted. The back-testing of past data raises the likelihood of a prosperous outcome.

An impetuous behaviour of a discretionary trader due to sudden changes in the market situations may end up in a loss. This may be due to the dearth of knowledge or failure to read the instability of the market. Methods like sentimental analysis help algorithmic traders to perform better in such situations and can read the variations in markets based on external factors. Technology is a part of the development and human beings have produced technologies that will define their century. Adapting to new and better methods of stock trading will help us achieve better results overall. One of the key reasons as to why algorithmic trading has become so admired is because of the benefit it has over traditional trading. Algorithmic trading provides better speed, reduced costs and accuracy compared to traditional trading methods for these reasons traditional traders should consider switching to algorithmic trading methods.

\section{CONCLUSION}

Algorithmic trading has introduced a new period for markets whose advantages are yet to be fully explored. Adjusting to new methods of stock trading can help to guarantee better results overall. Algorithmic trading is now a requirement for withstanding in the current stock market scenario because the future of stock trading tends to deal with automation and using Artificial intelligence-based stock trading models. According, to the recent reports algorithmic trading markets, is said to grow from 11.1 billion US dollars in 2019 to 18.8 billion US dollars in the year 2024, Increasing at a CAGR of 11.1 per- cent overall. Though algorithmic trading surpasses discretionary trading human intervention is still needed to some extent to ensure stability in the stock market. Most of the traditional traders are anxious and concerned that algorithmic trading will leave them behind and pose a threat to their businesses, but in fact, algorithmic trading can benefit traditional traders by increasing liquidity in the market and also makes easier the exit and entry processes. Using algorithmic trading can also help to considerably eliminate any price incapability's in the financial securities which are traded in the stock market. The chances on the arbitrage window are getting smaller day by day but with the introduction of algorithmic trading, the speed helps to find better arbitrage opportunities and helps to gain profit from them. There is a lot of prospective for algorithmic trading to thrive in India. Nevertheless, the future of algorithmic trading depends on how the rules of the country shape up. The rules in India have become resilient but algorithmic trading proceeds to demonstrate promising growth in the upcoming future. Researchers can father study on the profit earning capacity for both algorithmic trading and discretionary and find out which is a better and viable option for quantitative traders.

\section{REFERENCES}

1) 7 Reasons Statistical Arbitrage is important in Online Trading | executium Trading System. (2020). Retrieved 22 April 2020, from https://executium.com/trading-system/342139-7reasons-statistical-arbitrage-is-important-in-onlinetrading/

2) Algorithmic trading using technology to speed things up. (2020). Retrieved 21 April 2020, from https://www.tatateleservices.com/articles/algorithmic -trading-using-technology-to-speed-things-up

3) An Introduction to Momentum and Trend Trading. (2018). Retrieved 22 April 2020, from https://www.learntotrade.com.au/blog/news/anintroduction-to-momentum-and-trend-trading/

4) Arbitrage trading in forex explained. (2020). Retrieved 21 April 2020, from https://www.ig.com/en/trading-strategies/arbitragetrading-in-forex-explained-190621

5) Benefits of an arbitrage trading system | executium. (2020). Retrieved 22 April 2020, from https://executium.com/crypto-arbitrage/342190benefits-of-an-arbitrage-trading-system/.\%20 [Accessed: \%2007-\%20Apr-\%202020].

6) Bhagat, V. (2020). Candlestick patterns, anatomy and their significance. Retrieved 22 April 2020, from https://blog.quantinsti.com/candlestick-patternsmeaning/

7) Burns, S. (2020). Momentum Trading Vs. Trend Following. Retrieved 22 April 2020, from 


\section{International Journal of Engineering Applied Sciences and Technology, 2020 Vol. 4, Issue 12, ISSN No. 2455-2143, Pages 140-149 \\ Published Online April 2020 in IJEAST (http://www.ijeast.com)}

https://www.newtraderu.com/2014/07/28/momentum -trading-vs-trend-following/

8) Chen, J. (2019). Understanding the Theory of Mean Reversion. Retrieved 22 April 2020, from https://www.investopedia.com/terms/m/meanreversio n.asp

9) Decision Tree Classifier for Trading Part-1. (2017). Retrieved $21 \quad$ April 2020, from https://blog.quantinsti.com/decision-tree-classifiertrading-part-1/

10) Eastwood, G. (2017). Bloomberg - Are you a robot?. Retrieved $21 \quad$ April 2020, from https://www.bloomberg.com/professional/blog/3ways-big-data-changing-financial-trading/

11) Goldstein, M. (2014). Special Issue on Computerized and High-Frequency Trading: Guest Editor's Note. Financial Review, 49(2), 173-175. doi: 10.1111/fire. 12030

12) HENDERSHOTT, T., JONES, C., \& MENKVELD, A. (2011). Does Algorithmic Trading Improve Liquidity?. The Journal Of Finance, 66(1), 1-33. doi: 10.1111/j.1540-6261.2010.01624.x

13) JAIN, P. (2005). Financial Market Design and the Equity Premium: Electronic versus Floor Trading. The Journal Of Finance, 60(6), 2955-2985. doi: 10.1111/j.1540-6261.2005.00822.x

14) Japanese Candlesticks Charts | Benefits of Candlesticks Charts |FOREX.com. (2020). Retrieved 22 April 2020, from https://www.forex.com/en/education/educationthemes/technical-analysis/japanese-candlesticks/

15) Kuepper, J. (2018). How Statistical Arbitrage Can Lead to Big Profits. Retrieved 22 April 2020, from https://www.investopedia.com/articles/trading/07/stat istical-arbitrage.asp

16) Main Advantages of Fundamental and Technical Analysis | FXlinked. (2018). Retrieved 22 April 2020, from https://fxlinked.com/main-advantages-offundamental-and-technical-analysis/

17) Matthews, K. (2019). 5 Ways Data Science Is Changing Financial Trading. Retrieved 21 April 2020, from https://towardsdatascience.com/5-waysdata-science-is-changing-financial-tradingb7404d62ed6d

18) Maverick, J. (2019). How do I use software to make arbitrage trades?. Retrieved 21 April 2020, from https://www.investopedia.com/ask/answers/020415/h ow-do-i-use-software-make-arbitrage-trades.asp

19) Mitchell, C. (2020). Candlestick. Retrieved 22 April 2020 ,

from https://www.investopedia.com/terms/c/candlestick.as $\mathrm{p}$

20) Mitchell, C. (2020). How to Use a Moving Average to Buy Stocks. Retrieved 22 April 2020, from https://www.investopedia.com/articles/active- trading/052014/how-use-moving-average-buystocks.asp

21) Moy das, S. (2019). Algo Trading 101 for Dummies like Me. Retrieved 21 April 2020, from https://towardsdatascience.com/algo-trading-101-fordummies-like-me-b3938725d184

22) Online Trading | Forex, Spread Betting \& CFD Trading | City Index UK. (2020). Retrieved 22 April 2020, from https://www.cityindex.co.uk/

23) Retrieved 22 April 2020, from https://corporatefinanceinstitute.com/resources/knowl edge/trading-investing/momentum-indicators/

24) Singh, D. (2017). Moving Average Trading Strategies. Retrieved 22 April 2020, from https://blog.quantinsti.com/moving-average-tradingstrategies/

25) The best Forex indicators every trader should know. (2020). Retrieved 22 April 2020, from https://fbs.com/analytics/tips/technical-indicatorsevery-trader-should-know-6587

26) The Future of Algorithmic Trading. (2017). Retrieved 20 April 2020, from https://www.experfy.com/blog/the-future-ofalgorithmic-trading

27) Top Five Benefits of Technical Analysis in Currency Trading. (2020). Retrieved 22 April 2020, from http://www.traderslaboratory.com/forums/topic/9920 -top-five-benefits-of-technical-analysis-in-currencytrading/

28) Upadhyay, H. (2019). 7 benefits of mean reversion trading strategy - DYR. Retrieved 22 April 2020, from https://www.doubleyourreturns.in/why-youshould-trade-mean-reversion-trading-strategies/

29) W. Cheung, \& U. Kaymak. (2020). Retrieved 21 April 2020, from http://symorg.fon.bg.ac.rs/proceedings/2016/papers/B USINESS\%20ANALYTICS\%20AND\%20OPTIMIZ ATION.pdf

30) W. Cheung, \& U. Kaymak. (2020). Retrieved 21 April 2020, from http://symorg.fon.bg.ac.rs/proceedings/2016/papers/B USINESS\%20ANALYTICS\%20AND\%20OPTIMIZ ATION.pdf

31) What is the Role of Fuzzy Logic in Algorithmic Trading? - GeeksforGeeks. (2020). Retrieved 21 April 2020, from https://www.geeksforgeeks.org/what-is-the-role-offuzzy-logic-in-algorithmic-trading/

32) Yip, J. (2018). Algorithmic Trading using Sentiment Analysis on News Articles. Retrieved 21 April 2020, from https://towardsdatascience.com/httpstowardsdatascience-com-algorithmic-trading-usingsentiment-analysis-on-news-articles-83db77966704 